\title{
A method for estimating specimen tangent stiffness for hybrid simulation
}

\author{
Chung-Chan Hung and Sherif El-Tawil ${ }^{*} \dagger, \ddagger$ \\ Department of Civil and Environmental Engineering, University of Michigan, Ann Arbor, \\ MI 48109-2125, U.S.A.
}

\begin{abstract}
SUMMARY
Researchers have long recognized the importance and potential benefits of utilizing the tangent stiffness matrix of a test specimen in hybrid simulations employing implicit and mixed-integration schemes. However, the computation of the tangent stiffness matrix during testing has proved to be challenging, particularly for test specimens with more than one degree of freedom (dof). This paper presents a new methodology that is more straightforward and simpler than existing techniques for computing the tangent stiffness matrix of a multi-dof test specimen. The proposed method is combined with the operator-splitting method (OSM), and the capabilities, advantages and limitations of the new formulation are demonstrated through several examples. The accuracy, stability, and error propagation characteristics of the modified OSM are also studied theoretically as well as numerically. The research results show that the proposed algorithm provides results that are better than those produced via the regular OSM alone, especially for damped structures undergoing highly inelastic behavior during testing. Copyright (C) 2008 John Wiley \& Sons, Ltd.
\end{abstract}

Received 13 May 2008; Revised 21 July 2008; Accepted 22 July 2008

KEY WORDS: hybrid simulation; tangent stiffness; implicit methods; operator-splitting method; accuracy; stability

\section{INTRODUCTION}

The term hybrid simulation commonly refers to a class of methods that combine computational simulation with physical model testing to compute structural dynamic response. In its most general form, hybrid simulation could entail the combination of two or more purely computational models or multiple computational and physical models. In this method, a structure is numerically represented

\footnotetext{
${ }^{*}$ Correspondence to: Sherif El-Tawil, Department of Civil and Environmental Engineering, University of Michigan, Ann Arbor, MI 48109-2125, U.S.A.

†E-mail: eltawil@umich.edu

${ }^{\ddagger}$ Professor.
}

Contract/grant sponsor: National Science Foundation; contract/grant number: CMS 0530383

Contract/grant sponsor: University of Michigan

Copyright (C) 2008 John Wiley \& Sons, Ltd. 
as a discrete mass system and its time-history response to seismic excitation is computed by solving the differential equation of motion of the system. The difference between hybrid simulation and traditional numerical methods is that some of the terms of the restoring force vector in the differential equation of motion are either directly obtained by measurement from a physical test or computed from an associated computational model running in parallel with the main numerical time-integration scheme. When the entire restoring force vector is obtained from physical testing, hybrid simulation is commonly referred to as pseudo-dynamic testing. The pseudo-dynamic test technique was first proposed by Takanashi et al. in 1978 [1]. Since then, many pseudo-dynamic and hybrid testing algorithms have been proposed in [2-18]. These algorithms can be categorized as explicit, implicit or mixed algorithms.

The application of explicit algorithms for hybrid testing is limited by the intrinsic stability problem, which restricts the maximum time step that can be imposed. In essence, as the number of degrees of freedom (dofs) in a system increases, the size of the time step is bounded and controlled by the system's highest natural frequency. Exceeding the maximum allowable time step will lead to numerical instability and will eventually disrupt the simulation. As the limiting time step becomes smaller, the duration of a hybrid test employing an explicit scheme lengthens. While this results in additional costs and the inconvenience of a long test, a more serious concern is that the time step could become too small to permit a viable test. In such situations, the small time step results in displacement (or force) increments that are too small to reliably apply to a specimen.

Implicit methods, on the other hand, are unconditionally stable and are not hindered by the stability limitation. They are therefore attractive because they permit larger time steps to be taken. However, unless iterations are undertaken to ensure an acceptable level of accuracy, the use of large time steps can lead to an accumulation of errors, especially when nonlinear specimen response occurs. The need for iterations can be alleviated if an accurate tangent stiffness of the test structure is used in the simulations. Nevertheless, computing the tangent stiffness matrix for a multi-dof specimen has traditionally been considered practically difficult, and as a result, most existing implicit algorithms avoid using the tangent stiffness altogether. Instead, iterative corrections of the restoring force on the test specimen are sometimes used [15]. However, conducting iterations on a physical specimen is difficult to achieve in practice, especially for inelastic structures, and can lead to path-dependent effects. This difficulty has given rise to mixed explicit-implicit schemes where the objective is to compute an explicit target displacement to avoid iterations on the specimen during testing (e.g. the OS method proposed by Nakashima and Kato [19]).

As discussed next, a few researchers have recognized the importance and potential benefits of utilizing the tangent stiffness matrix of the test specimen in implicit and mixed-integration schemes and have taken steps to compute or update the tangent stiffness matrix during testing. They used the tangent stiffness for a variety of purposes including correction of the measured restoring force, suppressing displacement control errors, estimating energy errors in the pseudo-dynamic test and compensation for delay in real-time hybrid testing. This paper presents a new methodology that is more straightforward and simpler than existing techniques for computing the tangent stiffness matrix of a multi-dof test specimen.

\section{INTEGRATION TECHNIQUES FOR HYBRID TESTING}

Shing and Mahin [20,21] examined a number of explicit-integration techniques and recommended the use of a modified version of the Newmark algorithm, which includes frequency-proportional 
numerical damping. Based on a variation of Newmark's method by Hilbert et al. [22], Thewalt and Mahin [15,18] suggested an unconditionally stable implicit algorithm for pseudo-dynamic testing. In their method, estimation of structural stiffness is not required to get the restoring force at each time step. The restoring force is measured and corrected continuously within each time step by using the analogue feedback of the instantaneous restoring force associated with the input displacement. This algorithm has substantial advantages for testing structures with many dofs, where only a few modes contribute to the response. Since larger time steps are permitted by using this algorithm, the number of steps for a test is reduced, thus reducing the testing duration. Nevertheless, the actuator control accuracy remains unknown during testing, and thus the influence of the displacement control errors cannot be investigated. Moreover, additional hardware is needed to continuously correct the analog feedback.

To eliminate the drawbacks of the Thewalt and Mahin [15,18] algorithm, Shing et al. [23] developed an implicit algorithm also based on the implicit algorithm by Hilber et al. [22]. In their algorithm, they used a parameter to control the size of the displacement at each sub-step before a corrector displacement is applied to the physical specimen. This scheme produces every sub-step in a continuous loading/unloading form such that it prevents loading and unloading iterations on the physical specimen. Shing et al. [24] used this algorithm successfully to evaluate reinforced masonry shear wall components.

Pan et al. [14] developed a P2P internet online hybrid test system. In this new system, the equations of motion are not formulated for the entire model, but for each separate substructure instead. During the testing, two rounds of quasi-Newton iteration are used to ensure compatibility and equilibrium between each substructure. However, this scheme was not applied to structures that exhibit significant nonlinearity. Also, when the system includes more physical substructures, it could become more susceptible to experimental errors.

Nakashima et al. [25] proposed the operator-splitting method (OSM) for pseudo-dynamic testing. In this method, which is a mixed explicit-implicit scheme, an explicit target displacement is computed to avoid iterations on the specimen during testing. This is achieved by approximating the tangent stiffness of the tested structure by its initial stiffness in the restoring force calculations. The method is unconditionally stable when the initial stiffness of the tested structure is greater than the tangent stiffness during the entire test.

Combescure and Pegon [4] proposed a modification of OSM, which they termed the $\alpha$ operatorsplitting method ( $\alpha$-OSM). In this algorithm, the $\alpha$ parameter is introduced into the equations of motion to produce numerical dissipation that eliminates the spurious oscillation induced by control errors in the high-frequency modes. When $\alpha$ is set to zero, $\alpha$-OSM reverts into the regular OSM. The drawback of the method is that when a structure loses a large portion of its initial stiffness during testing, the results could become inaccurate [4]. In $\alpha$-OSM, the equation of motion of a structural system is expressed as

$$
\begin{aligned}
& M a_{n+1}+(1+\alpha) C v_{n+1}-\alpha C v_{n}+(1+\alpha) r_{n+1}-\alpha r_{n} \\
& \quad=(1+\alpha) f_{n+1}-\alpha f_{n}
\end{aligned}
$$

where $M$ is the mass matrix of the structure, $C$ is the damping coefficient matrix, $r$ is the restoring force vector, $a$ is the vector of nodal accelerations, $v$ is the vector of nodal velocities, $f$ is the vector of external excitation forces, subscript $n$ denotes the current time step. The displacement and velocity at the next time step are acquired from a Newmark-integration scheme. The obtained 
displacement and velocity are split into predicted and corrected responses

$$
\begin{gathered}
\tilde{d}_{n+1}=d_{n}+\Delta t v_{n}+\frac{\Delta t^{2}}{2}(1-2 \beta) a_{n} \\
\tilde{v}_{n+1}=v_{n}+\Delta t(1-\gamma) a_{n} \\
d_{n+1}=\tilde{d}_{n+1}+\Delta t^{2} \beta a_{n+1} \\
v_{n+1}=\tilde{v}_{n+1}+\Delta t \gamma a_{n+1}
\end{gathered}
$$

As previously discussed, the primary assumption of this method is that the restoring force at time step $n+1$ can be approximated as

$$
r_{n+1}\left(d_{n+1}\right) \approx \tilde{r}_{n+1}\left(\tilde{d}_{n+1}\right)+K^{I}\left(d_{n+1}-\tilde{d}_{n+1}\right)
$$

Substituting Equations (2)-(4) into Equation (1), the acceleration at time step $n+1$ can be computed as

$$
\begin{aligned}
a_{n+1}= & \hat{M}^{-1} \hat{f}_{n+1} \\
\hat{M}= & M+\gamma \Delta t(1+\alpha) C+\beta \Delta t^{2}(1+\alpha) K^{I} \\
\hat{f}_{n+1}= & (1+\alpha) f_{n+1}-\alpha f_{n}+\alpha \tilde{r}_{n}-(1+\alpha) \tilde{r}_{n+1}+\alpha C \tilde{v}_{n}-(1+\alpha) C \tilde{v}_{n+1} \\
& +\alpha\left(\gamma \Delta t C+\beta \Delta t^{2} K^{I}\right) a_{n}
\end{aligned}
$$

When the physical test structure becomes inelastic, the primary assumption of OSM and $\alpha$-OSM that the tangent stiffness of the specimen can be replaced with its initial stiffness (i.e. Equation (4)) becomes inaccurate, and can adversely affect the accuracy of the test method.

A few researchers have taken steps to compute and incorporate the tangent stiffness matrix during testing. Efforts appear to be limited because of the practical difficulty of directly estimating the tangent stiffness as the test progresses. One of the earliest attempts to employ the tangent stiffness during testing was by Nakashima and Kato [19]. They estimated the tangent stiffness in an effort to correct the measured restoring force and suppress displacement control errors. In their work, they collected the necessary information from sub-steps within a time step to estimate the tangent stiffness. Nevertheless, they only used the estimated tangent stiffness to modify the restoring force instead of merging the estimated tangent stiffness with the integration algorithm as is done in this paper.

Thewalt and Roman [26] developed an approach based on the BFGS formula [27] to estimate the tangent stiffness during testing. They used the estimated tangent stiffness to estimate energy errors in the pseudo-dynamic test. Carrion and Spencer [28] estimated the tangent stiffness based on the Broyden formula [29]. They used the estimated tangent stiffness for compensation of delay in real-time hybrid testing.

In this paper, a new strategy for estimating the tangent stiffness is proposed. The scheme is general in that it can be applied to a variety of implicit and mixed-integration methods. However, in this paper, the new methodology is combined with the traditional OSM to show the resulting advantages of implementing it within a hybrid simulation framework. These advantages are demonstrated using a series of hybrid simulation exercizes, including test structures that exhibit significant inelastic behavior and which have multiple dofs. 


\section{PROPOSED OPERATOR-SPLITTING METHOD WITH UPDATED TANGENT STIFFNESS (OSM-US)}

The incremental force and displacement at time step $n+1$ can be associated through the tangent stiffness at time step $n+1$ as

$$
\left\{\begin{array}{c}
\Delta \tilde{r}_{n+1}^{(1)} \\
\Delta \tilde{r}_{n+1}^{(2)} \\
\vdots \\
\vdots \\
\Delta \tilde{r}_{n+1}^{\text {dof }}
\end{array}\right\}_{\text {dof } \times 1}=\left[\begin{array}{ccccc}
K_{n+1}^{1,1} & K_{n+1}^{1,2} & \ldots & \ldots & K_{n+1}^{1, \text { dof }} \\
K_{n+1}^{2,1} & K_{n+1}^{2,2} & & & K_{n+1}^{2, \text { dof }} \\
\vdots & \vdots & & & \vdots \\
\vdots & \vdots & & & \vdots \\
K_{n+1}^{\text {dof,1 }} & K_{n+1}^{\text {dof,2 }} & \ldots & \ldots & K_{n+1}^{\text {dof,dof }}
\end{array}\right]_{\text {dof } \times \text { dof }}\left\{\begin{array}{c}
\Delta \tilde{d}_{n+1}^{(1)} \\
\Delta \tilde{d}_{n+1}^{(2)} \\
\vdots \\
\vdots \\
\Delta \tilde{d}_{n+1}^{\text {(dof }}
\end{array}\right\}_{\text {dof } \times 1}
$$

where $\Delta \tilde{r}_{n+1}^{(q)}$ is the incremental restoring force at the $q$ th dof of the model at time step $n+1$. $K_{n+1}^{x, y}$ is the component value at $(x, y)$ position of the tangent stiffness matrix at time step $n+1$. $\Delta \tilde{d}_{n+1}^{(q)}$ is the incremental predicted displacement at the $q$ th dof of the model at time step $n+1$.

By assuming that the tangent stiffness does not change substantially during the current and previous $m-1$ steps, i.e. for a total of $m$ steps from time step $n+2-m$ to $n+1$, the relation between the incremental force and the incremental displacement during the time interval $n+2-m$ to $n+1$ can be expressed as

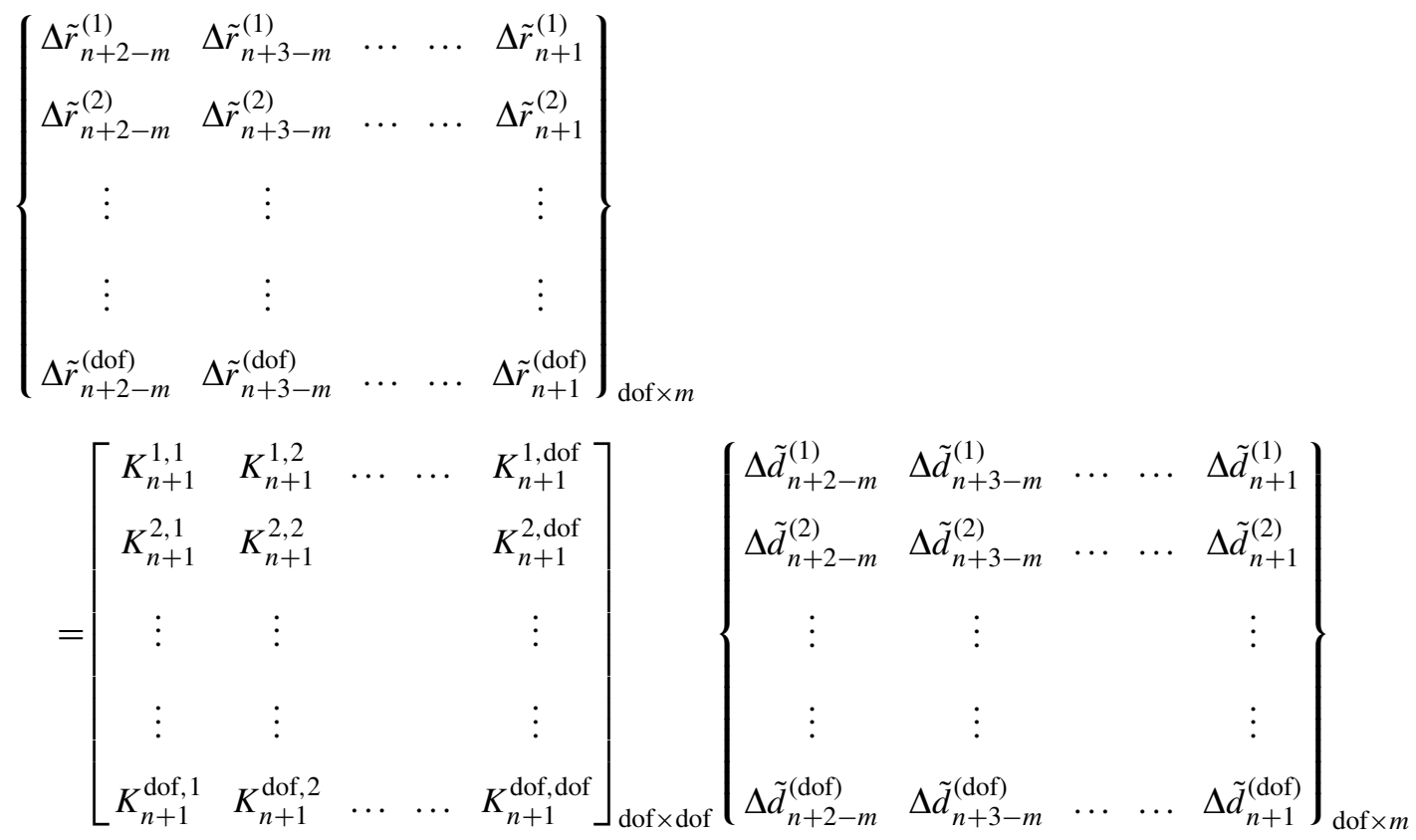


Transposing Equation (7),

$$
\begin{aligned}
& \left\{\begin{array}{ccccc}
\Delta \tilde{r}_{n+2-m}^{(1)} & \Delta \tilde{r}_{n+2-m}^{(2)} & \ldots & \ldots & \Delta \tilde{r}_{n+2-m}^{(\mathrm{dof})} \\
\Delta \tilde{r}_{n+3-m}^{(1)} & \Delta \tilde{r}_{n+3-m}^{(2)} & \ldots & \ldots & \Delta \tilde{r}_{n+3-m}^{(\mathrm{dof})} \\
\vdots & \vdots & & & \vdots \\
\vdots & \vdots & & & \vdots \\
\Delta \tilde{r}_{n+1}^{(1)} & \Delta \tilde{r}_{n+1}^{(2)} & \ldots & \ldots & \Delta \tilde{r}_{n+1}^{(\mathrm{dof})}
\end{array}\right\} \\
& =\left[\begin{array}{ccccc}
\Delta \tilde{d}_{n+2-m}^{(1)} & \Delta \tilde{d}_{n+2-m}^{(2)} & \ldots & \ldots & \Delta \tilde{d}_{n+2-m}^{\text {(dof) }} \\
\Delta \tilde{d}_{n+3-m}^{(1)} & \Delta \tilde{d}_{n+3-m}^{(2)} & \ldots & \ldots & \Delta \tilde{d}_{n+3-m}^{\text {(dof) }} \\
\vdots & \vdots & & & \vdots \\
\vdots & \vdots & & & \vdots \\
\Delta \tilde{d}_{n+1}^{(1)} & \Delta \tilde{d}_{n+1}^{(2)} & \ldots & \ldots & \Delta \tilde{d}_{n+1}^{\text {dof }}
\end{array}\right]\left\{\begin{array}{ccccc}
K_{n+1}^{1,1} & K_{n+1}^{2,1} & \ldots & \ldots & K_{n+1}^{\text {dof, } 1} \\
K_{n+1}^{1,2} & K_{n+1}^{2,2} & & & K_{n+1}^{\text {dof, }} \\
\vdots & \vdots & & \vdots \\
\vdots & \vdots & & \vdots \\
K_{n+1}^{1, \text { dof }} & K_{n+1}^{2, \text { dof }} & \ldots & \ldots & K_{n+1}^{\text {dof,dof }}
\end{array}\right\}
\end{aligned}
$$

For Equation (8) to yield a solution, the number of considered time steps, $m$, must be equal to or larger than the number of dof in the test structure, i.e. $m \geqslant$ dof. Equation (8) can be partitioned into dof equations as shown in below

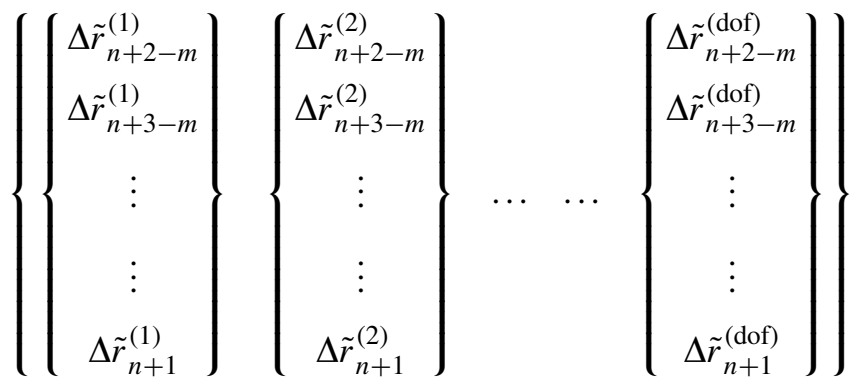

$$
\begin{aligned}
& =\left[\begin{array}{ccccc}
\Delta \tilde{d}_{n+2-m}^{(1)} & \Delta \tilde{d}_{n+2-m}^{(2)} & \ldots & \ldots & \Delta \tilde{d}_{n+2-m}^{(\mathrm{dof})} \\
\Delta \tilde{d}_{n+3-m}^{(1)} & \Delta \tilde{d}_{n+3-m}^{(2)} & \ldots & \ldots & \Delta \tilde{d}_{n+3-m}^{(\mathrm{dof})} \\
\vdots & \vdots & & & \vdots \\
\vdots & \vdots & & & \vdots \\
\Delta \tilde{d}_{n+1}^{(1)} & \Delta \tilde{d}_{n+1}^{(2)} & \ldots & \ldots & \Delta \tilde{d}_{n+1}^{(\mathrm{dof})}
\end{array}\right]
\end{aligned}
$$




$$
\times\left\{\begin{array}{c}
K_{n+1}^{1,1} \\
K_{n+1}^{1,2} \\
\vdots \\
\vdots \\
K_{n+1}^{1, \text { dof }}
\end{array}\right\}\left\{\begin{array}{c}
K_{n+1}^{2,1} \\
K_{n+1}^{2,2} \\
\vdots \\
\vdots \\
K_{n+1}^{2, \text { dof }}
\end{array}\right\} \cdots \cdots
$$

When $m=$ dof in Equation (9), a unique solution can be found for the tangent stiffness coefficients. However, when $m>$ dof in Equation (9), the system becomes over determined, and the least-squares method, for example, as implemented in [30], can be used to solve for the tangent stiffness matrix.

The initial stiffness in the regular OSM and $\alpha$-OSM (as shown in Equation (4)) can now be replaced by the estimated tangent stiffness computed from Equation (9). The restoring force at time step $n+1$ is now approximated as

$$
r_{n+1}\left(d_{n+1}\right) \approx \tilde{r}_{n+1}\left(\tilde{d}_{n+1}\right)+K_{n+1}^{\mathrm{T}}\left(d_{n+1}-\tilde{d}_{n+1}\right)
$$

where $K_{n+1}^{\mathrm{T}}$ is the tangent stiffness at time step $n+1$. As shown in Figure 1, the restoring force $r_{n+1}\left(d_{n+1}\right)$ is an approximation of the sum of the predicted restoring force $\tilde{r}_{n+1}\left(\tilde{d}_{n+1}\right)$, which is the feedback from the substructures subjected to the predicted displacement, and a modification term, $K_{n+1}^{\mathrm{T}}\left(d_{n+1}-\tilde{d}_{n+1}\right)$, which is the difference between the predicted and the actual displacement multiplied by the tangent stiffness. Equivalent force equilibrium using the tangent stiffness can now be expressed as

$$
\begin{aligned}
\hat{M}_{n+1} a_{n+1} & =\hat{f}_{n+1} \\
\hat{M}_{n+1} & =M+\frac{\Delta t C_{n+1}}{2}+\frac{\Delta t^{2} K_{n+1}^{\mathrm{T}}}{4} \\
\hat{f}_{n+1} & =f_{n+1}-\tilde{r}_{n+1}-C_{n+1} \tilde{v}_{n+1}
\end{aligned}
$$

The procedure for hybrid simulation using OSM-US is illustrated in Figure 2. The predicted displacement and velocity are first computed based on the response from the previous time step. After the predicted displacements are imposed upon the test specimen, the resulting reaction forces, $\tilde{r}_{n+1}$, and the actual imposed displacements, $\tilde{d}_{n+1}^{m}$, are measured. Then, the incremental values of the restoring forces and the measured displacements from the current and previous $m-1$ steps are used to calculate the tangent stiffness matrix. The damping coefficient, equivalent force and equivalent mass are then updated using the tangent stiffness. After that, the acceleration, the corrected displacement and the velocity of the current time step are computed from Equations (3) and (11), and are used for the next time step.

Two rules are imposed on the algorithm to ensure reasonable results. The first resets the reloading/unloading tangent stiffness to the initial stiffness right after the point where the displacement direction changes. After reloading/unloading at this point, the original scheme continues collecting the previous records and estimating the stiffness. This rule limits the proposed method to structures dominated by first mode response, where unloading of one member is associated with unloading of the entire structure. The second rule is more practical in nature. It filters out 


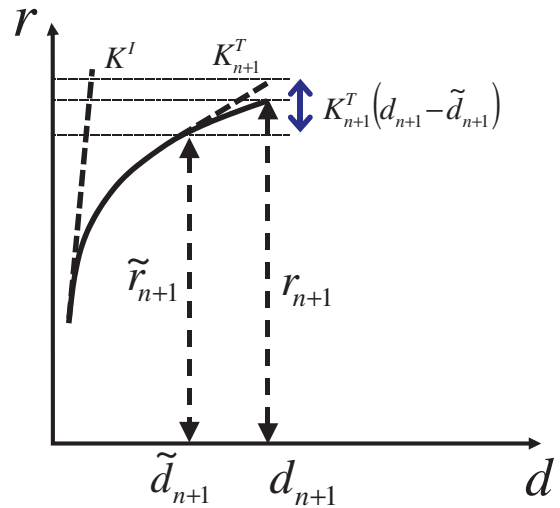

Figure 1. The approximated restoring force at time step $n+1$.

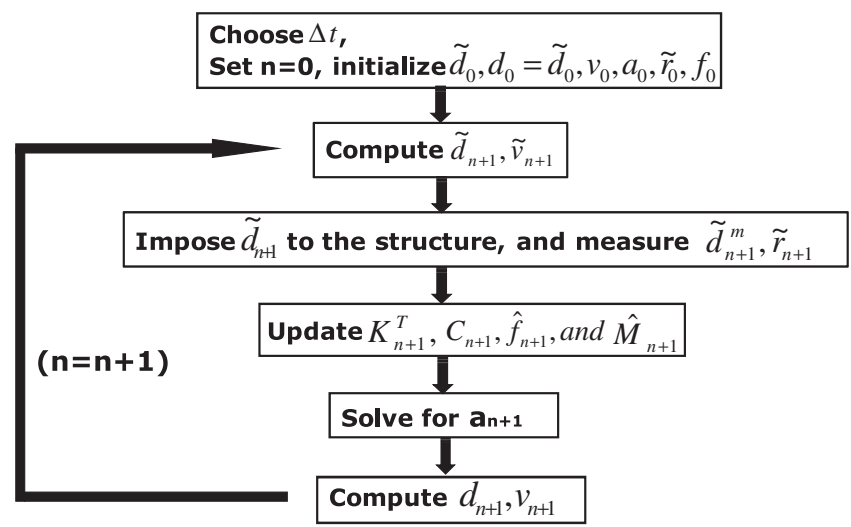

Figure 2. Flowchart of OSM-US operations.

the steps where the imposed displacements are smaller than a user-specified threshold, specified as a minimum incremental displacement. This is to ensure that only steps that are large enough to contribute reliably to the stiffness calculations are utilized in the computations. Although user experience clearly plays a role in identifying this parameter, a number that is two to three times the displacement resolution of the actuator is recommended.

\section{NUMERICAL SIMULATION OF HYBRID TESTING USING OSM-US}

As discussed above and in $[4,25]$, the use of the traditional OSM for pseudo-dynamic testing could generate unreliable results, especially for structures whose stiffness degrades significantly during testing. The new OSM-US methodology proposed herein alleviates this problem. To highlight the advantages of OSM-US, four numerical models that are designed to exhibit significant inelastic 
behavior during hybrid simulation are used. The simulations are conducted without damping and with 3\% Rayleigh damping. As shown in Figure 3(a), Model 1 represents a 1-story, 1 dof, structure. Model 2 (Figure 3(b)) represents a 2-story structure with 2 lateral dofs. Model 3 (Figure 3(c)) represents a 6-story structure with 6 lateral dofs. In Model 4, a 2-story structure is split into two substructures (Figure 3(d)). The bottom substructure, which would usually be physically tested in a hybrid simulation, is numerically simulated here, while the top column is treated as a numerical substructure.

The cross section of the column used in all examples is shown in Figure 4. OpenSees [31] is used to model the inelastic behavior of the members. Concrete02 and Steel01 material models in OpenSees are used to represent concrete and steel, respectively. Parameter definitions and properties of the material models are shown in Figure 5 and Table I. The 1940 El Centro earthquake history record with $\mathrm{PGA}=0.36 \mathrm{~g}$ is used as the external excitation throughout this study.

Lateral displacement control for Model 1 (Figure 3(a)) is achieved using the pseudo-dynamic method with both OSM and OSM-US. The results are compared together along with the reference results from OpenSees in Figure 6. A comparison of the displacement responses in Figure 6(a) shows that OSM deviates a little from the reference solution, especially at the peaks, while OSMUS consistently matches the results well. The computed hysteresis responses are shown in Figure 6(b), which shows that the simulated solution from OSM-US matches the reference solution better than that from OSM, especially when the model experiences significant inelastic behavior.

(a)

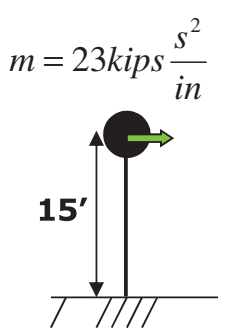

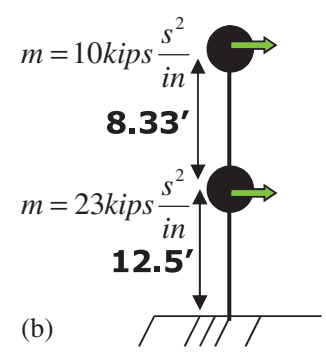

(b)

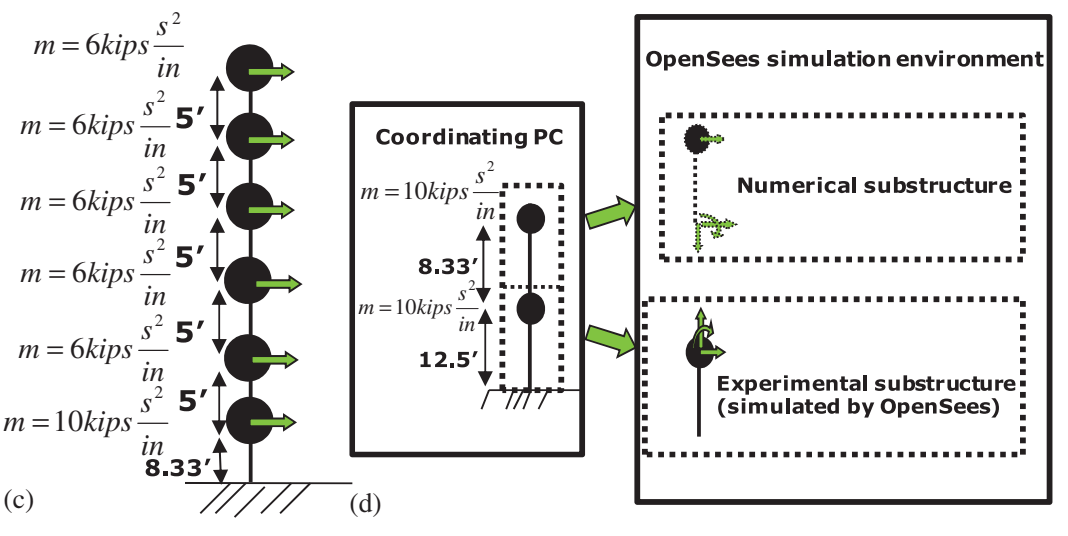

(c)

Figure 3. Demonstration examples of the proposed OSM-US: (a) Model 1; (b) Model 2; (c) Model 3; and (d) Model 4. 


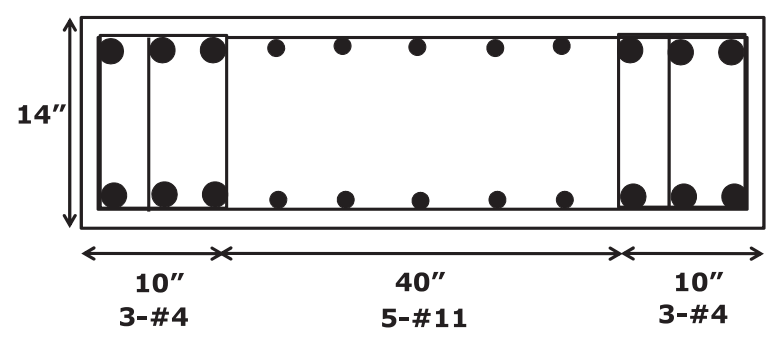

Figure 4. Cross section of the column used in the examples.
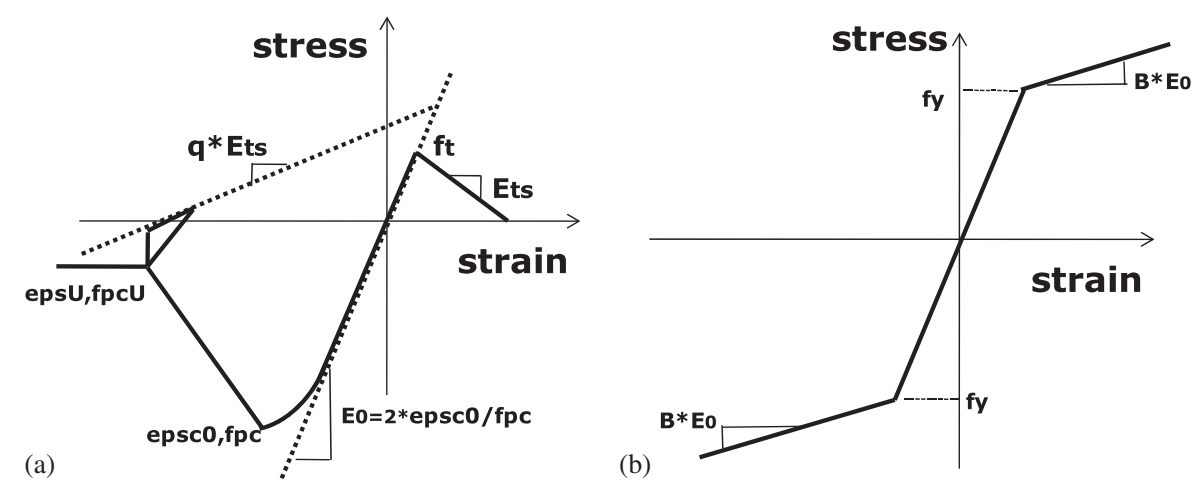

Figure 5. Concrete and steel bar material models in OpenSees: (a) concrete02 model and its parameters and (b) material01 model and its parameters.

Table I. Material parameters of the concrete and steel models.

\begin{tabular}{lccccccc}
\hline Concrete properties & $f p c(k s i)$ & epsc0 & $f p c u(k s i)$ & epsU & $q$ & $f t(k s i)$ & Ets(ksi) \\
\hline Unconfined concrete & -6 & -0.002 & -0.4 & -0.01 & 0.1 & 0.6 & 300 \\
Confined concrete & -7.2 & -0.0045 & -2.4 & -0.03 & 0.1 & 0.72 & 360 \\
& $f y(k s i)$ & $E 0(k s i)$ & $B$ & & & & \\
Steel bar properties & 60 & 29000 & 0.01 & & & & \\
\hline
\end{tabular}

The behavior of Model 2 (Figure 3(b)) also demonstrates the capabilities of OSM-US. Simulation of the undamped model is conducted with an incremental time step $0.005 \mathrm{~s}$ and $m=3$. The resulting displacement responses from OpenSees, OSM and OSM-US are compared in Figure 7, which indicates that both the integration methods appear to match the reference results from OpenSees quite well. However, as shown in Figure $7(\mathrm{~b})$, the cumulative energy error, $E_{\mathrm{c}}$, computed for the top-node response, shows that the error from OSM-US is less than half of the result computed 

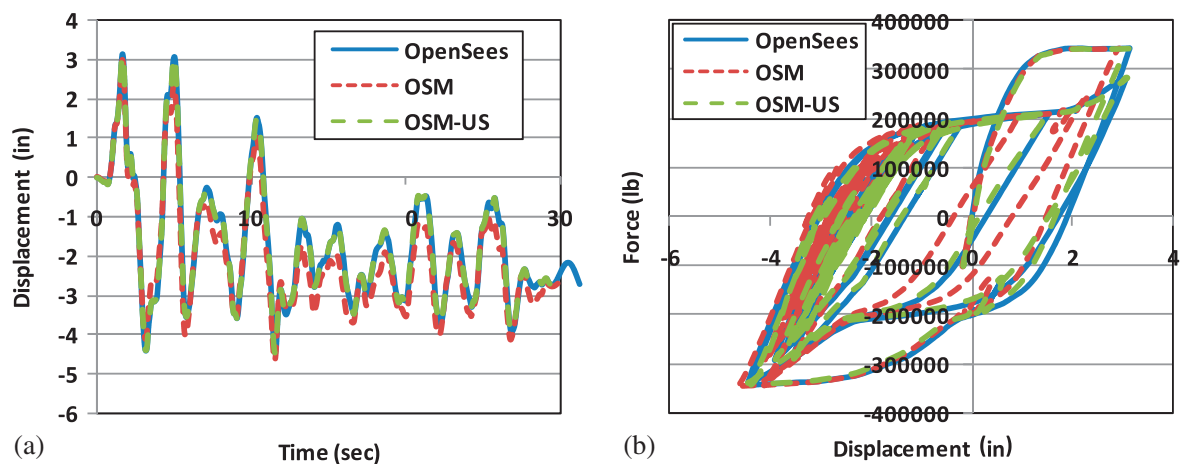

Figure 6. Simulated responses for Model 1: (a) displacement of top node and (b) hysteresis response.
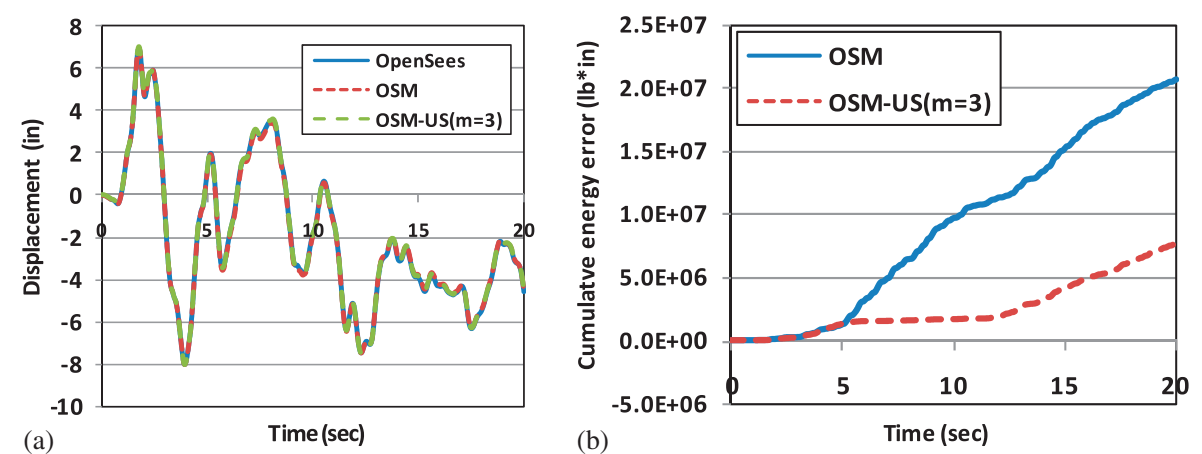

Figure 7. Simulated top-node responses of undamped Model 2: (a) displacement response of top node and (b) cumulative energy index.

from OSM. The cumulative energy error is defined as

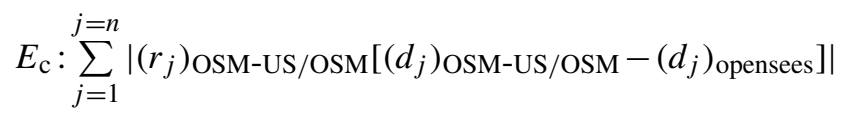

$\left(d_{j}\right)_{\text {MOSM/OSM }}$ is the displacement of the node of interest computed from OSM-US or OSM, $\left(d_{j}\right)_{\text {opensees }}$ is the displacement of the node of interest from OpenSees, $\left(r_{j}\right)_{\text {MOSM/OSM }}$ is the restoring force at the same node from OSM-US or OSM, and $n$ is the current time step.

The same model (Model 2), but with 3\% damping, more clearly demonstrates the advantages of OSM-US over OSM. It is obvious from Figure 8 that the resulting displacement from OSM deviates from the reference OpenSees solution, whereas the OSM-US results remain very good. The success of OSM-US in this case is due to its ability to utilize the correct tangent stiffness matrix in the Rayleigh damping computations.

Model 3 (Figure 3(c)) is simulated with a time step of $0.005 \mathrm{~s}$ and $m=8$. Figure 9(a) shows the response of the undamped model. Again, though the differences between OSM and OSM-US are 


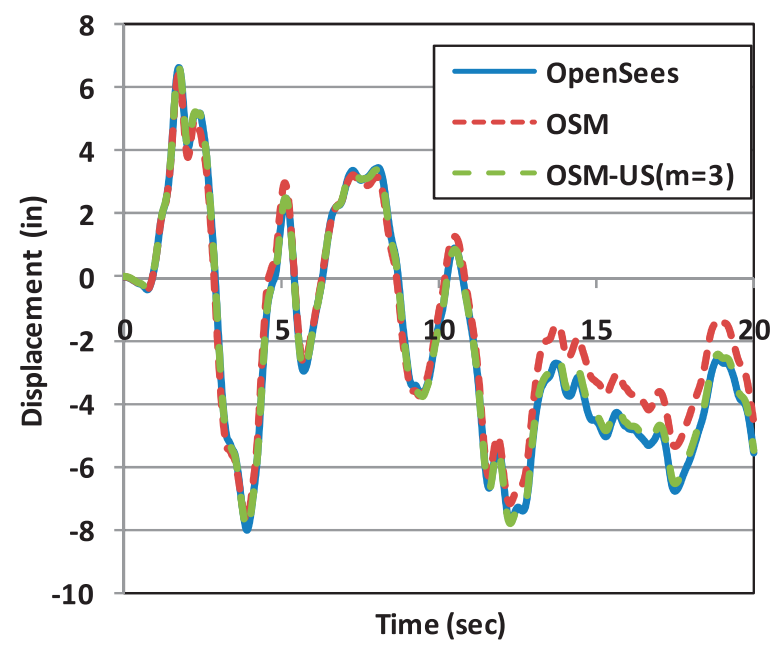

Figure 8. Computed top-node response of Model 2 (damped).
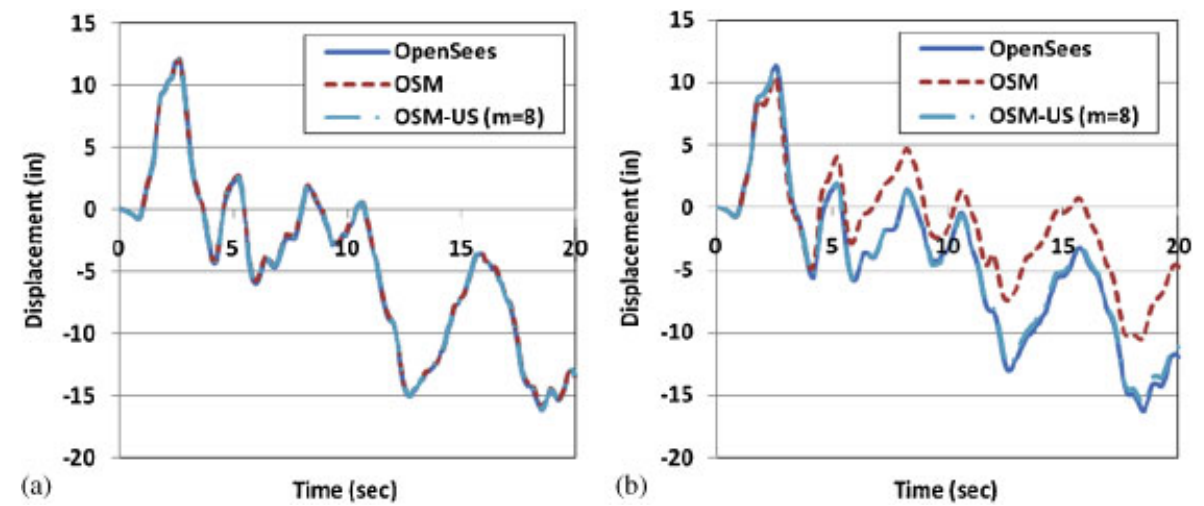

Figure 9. Simulated top-node responses of Model 3: (a) undamped response and (b) damped response.

not obvious in this figure, $E_{\mathrm{c}}$ computed at the top node from OSM-US is significantly less than that for OSM, as observed in Model 2. Also, as observed in Model 2, differences in displacement response become more pronounced when damping is accounted for (Figure 9(b)). In this case OSM-US is much closer to the reference solution than OSM.

The influence of $m$ on the simulation results is shown in Figure 10. Figure 10(a) shows $E_{\mathrm{c}}$ computed at the top node. It is observed that the cumulative energy becomes larger as $m$ increases. Recall that the minimum value for $m$ is the number of dofs. At $m=80$, the cumulated energy error from OSM-US is close to that from OSM. Figure 10(b) indicates that the disagreement between the displacement response from OSM-US and the reference solution becomes larger as $m$ continues to increase. 

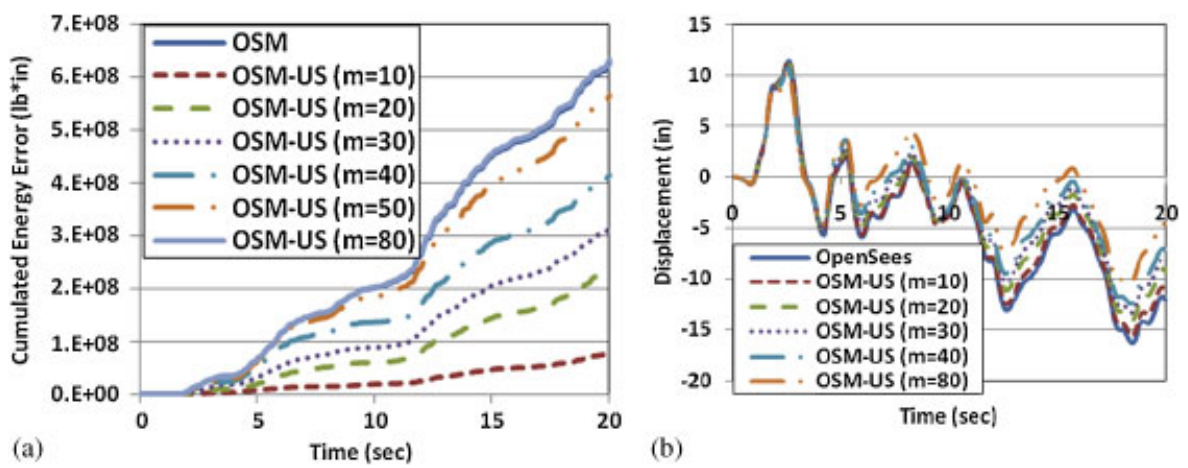

(b)

Figure 10. Effect of $m$ parameter on the response of Model 3: (a) cumulative energy error and (b) displacement of the top node.

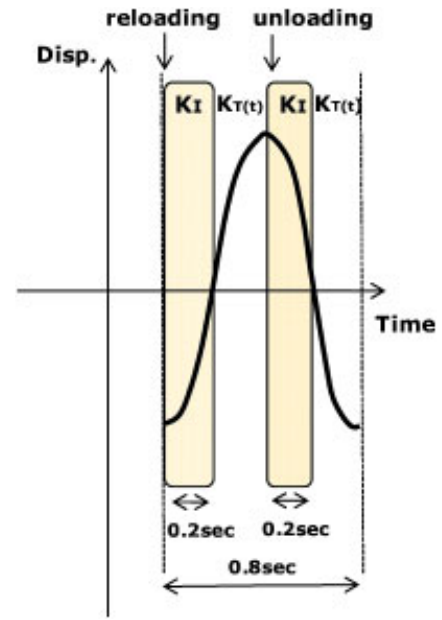

Figure 11. The approximate distribution of the stiffness for the 2 nd mode response.

To better understand the influence of $m$ and why accuracy degrades as $m$ increases beyond a certain limit, consider that the 1st and 2nd mode periods for Example 3 are 4.9 and $0.8 \mathrm{~s}$, respectively. When $m=40$, the imposed displacements and reaction forces from the past 40 time steps, corresponding to a duration of $0.2 \mathrm{~s}$ in this case, are used to estimate the current tangent stiffness. In other words, immediately after the point of unloading/reloading, the proposed scheme uses the initial stiffness for integration for $0.2 \mathrm{~s}$, a period during which significant inelasticity occurs. Figure 11 demonstrates this idea and shows that for a structure vibrating solely in 2 nd mode, the $0.2 \mathrm{~s}$ is too large a fraction of the 2 nd mode period to allow realistic updating of the stiffness properties. Clearly, the results will continue to deteriorate as $m$ increases.

The trends observed in Models 1-3 are also observed in Model 4, which is solved using $m=8$ and a smaller time step of $0.002 \mathrm{~s}$ due to the high axial stiffness of the column. 


\section{ERROR PROPAGATION CHARACTERISTICS OF OSM-US}

Actuator control errors occur during a hybrid test with a physical component. In order to gauge the effect of displacement control errors on the proposed OSM-US, numerical simulation of a hybrid experiment with induced displacement control errors is conducted. Model 2, Figure 3(b), is selected for this purpose. To simulate the effect of control errors, a random displacement control error signal with an average of 0 and a variance of $3 \mathrm{E}-7$ inch is introduced. The resulting maximum control error is $1.75 \mathrm{E}-3$ inch, which is within the range of accuracy of commercially available actuators. The simulation result is shown in Figure 12 and compared with the results from OpenSees (without induced errors). It can be observed from Figure 12 that the effect of the induced displacement control errors on the resulting response from OSM-US is negligible for this particular case. Nevertheless, if the error propagation induced by actuator control errors is considered to be too deleterious, the T-Modification strategy suggested by Nakashima and Kato [19] can be applied to mitigate displacement control errors. The T-Modification approach is only possible in this case because the tangent stiffness matrix is available through the OSM-US algorithm.

Compatibility between substructures in hybrid simulation is assured through displacement control of the dofs at the boundaries between experimental and numerical substructures. However, equilibrium, i.e. the presence of unbalanced forces, at the boundaries between both the substructures is not necessarily assured unless iterations are performed. Since OSM-US, which employs a specimen's tangent stiffness, traces the nonlinear response better than OSM, which utilizes the initial stiffness, it is expected that unbalanced forces will be reduced at the interface.

To investigate the growth of unbalanced forces in both OSM and OSM-US, hybrid simulation involving Model 4 is used. Unbalanced moments at the interface are measured through a normalized quantity, $\left|M_{\mathrm{t}}+M_{\mathrm{b}} / M_{\max }\right|$, where $M_{\mathrm{t}}$ is the moment at the boundary node from top element, $M_{\mathrm{b}}$ is the moment at the boundary node from the bottom element, $M_{\max }$ is the maximum moment in both the elements during the simulation. The result is extracted and plotted for a portion of response as shown in Figure 13. It can be seen from the linear regression of the unbalanced force that the OSM-US has significantly reduced unbalanced forces compared with OSM. It is noted

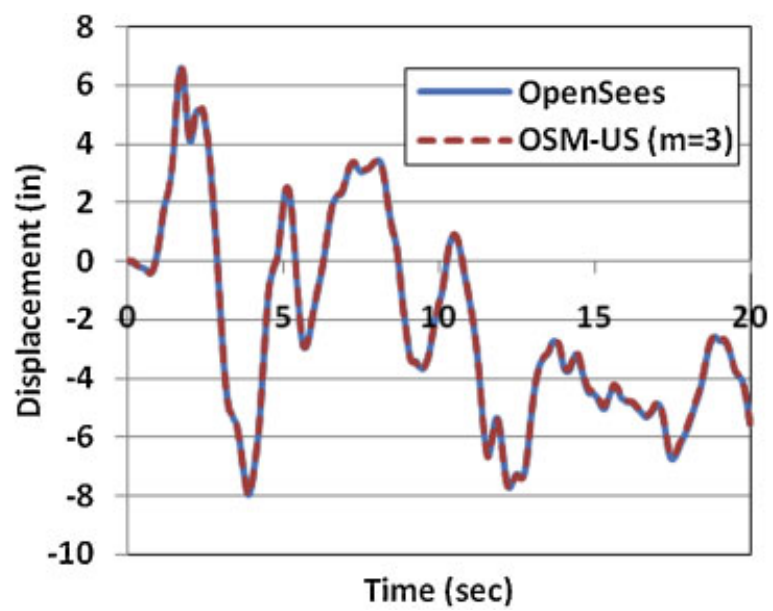

Figure 12. Effect of induced displacement control errors on the response of top node in Model 2. 


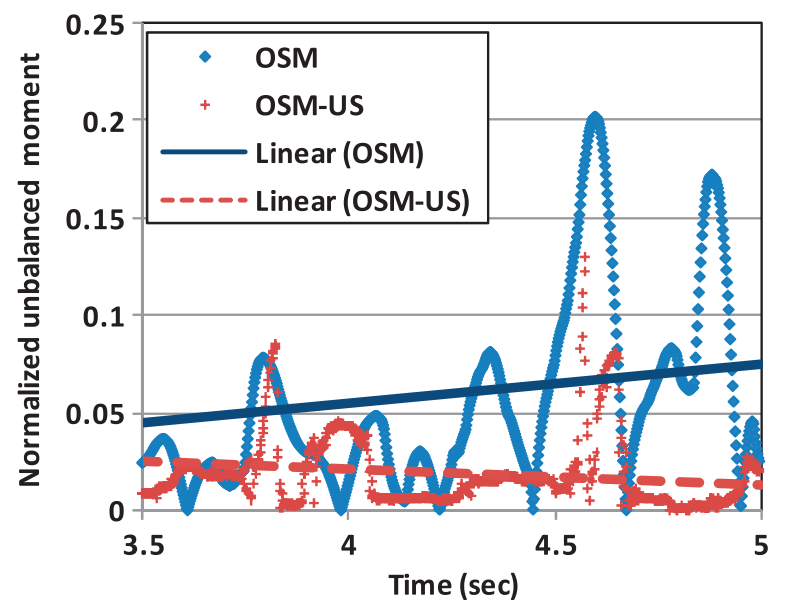

Figure 13. Normalized unbalanced moment between substructures in Model 4.

that the larger unbalanced force segments in the OSM-US correspond to the portion where the initial tangent stiffness is used for integration. This result suggests that OSM-US can improve the quality of the solution with fewer iterations if iterations are deemed necessary.

\section{STABILITY AND ACCURACY CHARACTERISTICS OF OSM-US}

When OSM-US is applied to hybrid simulation, the equations of motion for an elastic, single dof system can be written as

$$
M a_{n+1}+\left[C^{\mathrm{EST}}\left(v_{n+1}-\tilde{v}_{n+1}\right)+C^{\mathrm{R}}\left(\tilde{v}_{n+1}\right)\right]+\left[K^{\mathrm{EST}}\left(d_{n+1}-\tilde{d}_{n+1}\right)+K^{\mathrm{R}}\left(\tilde{d}_{n+1}\right)\right]=F_{n+1}
$$

where $C^{\mathrm{EST}}$ and $K^{\mathrm{EST}}$ are the estimated damping coefficient and tangent stiffness, and $C^{\mathrm{R}}$ and $K^{\mathrm{R}}$ are the real damping coefficient and tangent stiffness, respectively. Equation (13) can be further rearranged in a recursive form as shown in below

$$
X_{n+1}=A X_{n}
$$

where

$$
X_{n}=\left\{\begin{array}{c}
\tilde{d}_{n} \\
\tilde{v}_{n} \Delta t \\
a_{n} \Delta t^{2}
\end{array}\right\}, \quad A=\left[\begin{array}{ccc}
1 & 1 & 1 \\
0 & 1 & 1 \\
P & Q & Q
\end{array}\right]
$$

is the amplification matrix,

$$
P=\frac{-\Omega^{2}}{1+\beta \xi \Omega+\frac{1}{4} \alpha \Omega^{2}}, \quad Q=\frac{-2 \Omega \xi-\Omega^{2}}{1+\beta \xi \Omega+\frac{1}{4} \alpha \Omega^{2}}, \quad \xi=\frac{C^{\mathrm{R}}}{2 M \omega} \quad \text { and } \quad \Omega=\omega \Delta t=\sqrt{\frac{K^{\mathrm{R}}}{M}} \Delta t
$$


$\alpha$ is defined as the estimation error of the tangent stiffness and is expressed as $\alpha=K^{\mathrm{EST}} / K^{\mathrm{R}}, \beta$ is defined as the estimation error of the damping and is expressed as $\beta=C^{\mathrm{EST}} / C^{\mathrm{R}}$.

The stability and accuracy of OSM-US can be investigated through the characteristics of the matrix A [22]. The spectral radius, $\rho(A)$, is the maximum absolute eigenvalue of $A$. The algorithm is stable when $\rho(A) \leqslant 1$. With $\alpha=\beta=1$, the spectral radius versus $\Omega$ with varying $\xi$ can be plotted as shown in Figure 14. It can be seen that the stability of OSM-US increases (i.e. spectral radius reduces) as $\xi$ increases.

The effect of the accuracy of the computed tangent stiffness on the stability can also be investigated. In this case, $\xi$ is fixed at $2 \%$, and $\beta=1$. The result is plotted in Figure 15(a). It can be seen from the figure that the stability drops as the accuracy of the estimated tangent stiffness decreases. By fixing $\alpha=1$ and $\xi=2 \%$, the relationship between the spectral radius versus $\Omega$ with varying $\beta$ is plotted in Figure 15(b). It shows that stability increases with a lower $\beta$, but the influence of $\beta$ on stability is not as obvious as that of $\alpha$.

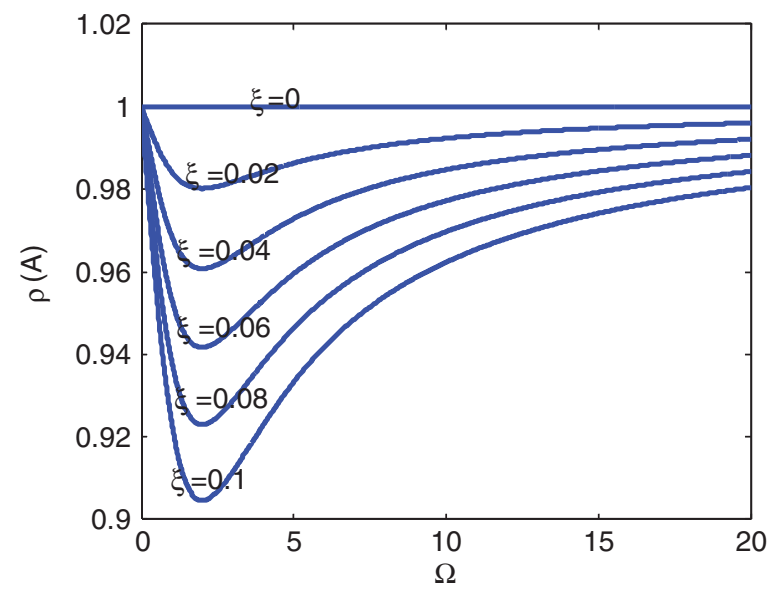

Figure 14. Trends in the spectral radius with varying $\Omega$ and $\xi(\alpha=\beta=1)$.
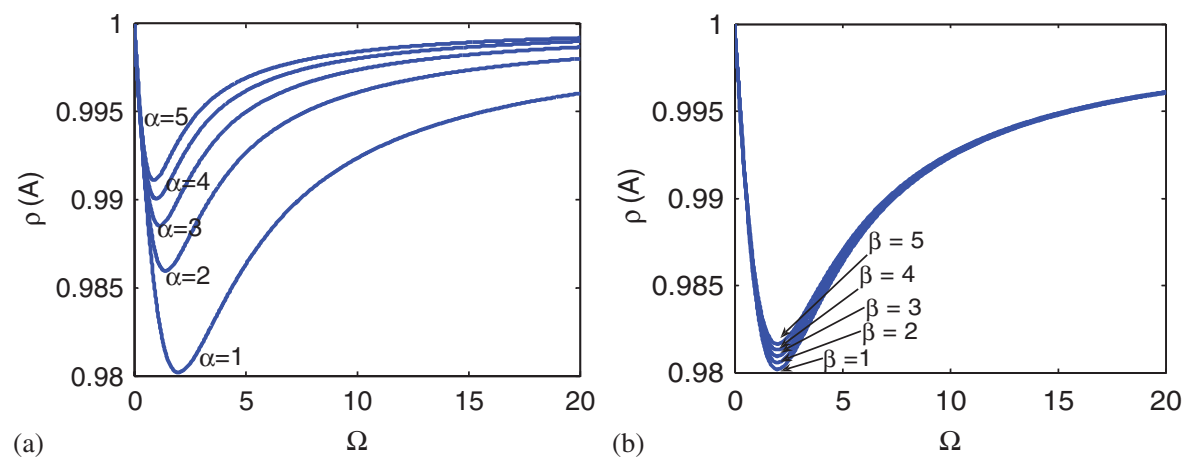

(a)

$\Omega$

(b)

Figure 15. Trends in the spectral radius with varying $\Omega$ : (a) $\Omega$ with varying $\alpha(\beta=1, \xi=0.02)$ and (b) $\Omega$ with varying $\beta(\alpha=1, \xi=0.02)$. 
The numerical accuracy of OSM-US is related to the ratio of period distortion and numerical damping $[10,32]$. The non-zero eigenvalues of $A$ can be expressed as

$$
\lambda_{1,2}=\exp [(-\hat{\xi} \pm \mathrm{i}) \bar{\Omega}]=L_{1} \pm \mathrm{i} L_{2}
$$

where

$$
\hat{\xi}=-\frac{\ln \left(L_{1}^{2}+L_{2}^{2}\right)}{2 \bar{\Omega}}, \quad \bar{\Omega}=\tan ^{-1}\left(\frac{L_{2}}{L_{1}}\right)
$$

Also, the displacement solution for a viscously underdamped free-vibration response can be written as [10]

$$
D_{n}=\exp \left(-\xi^{\prime} \bar{\omega}_{n} n \Delta t\right)\left[c_{1} \cos \left(\bar{\omega}_{d} n \Delta t\right)+c_{2} \sin \left(\bar{\omega}_{d} n \Delta t\right)\right]
$$

where

$$
\xi^{\prime}=\frac{\hat{\xi}}{\sqrt{1+\hat{\xi}^{2}}}, \quad \bar{\omega}_{d}=\frac{\bar{\Omega}}{\Delta t} \quad \text { and } \quad \bar{\omega}_{n}=\frac{\bar{\omega}_{d}}{\sqrt{1-\xi^{2}}}
$$

The numerical damping is defined as

$$
\bar{\xi}=\xi^{\prime}-\xi
$$

In the case of $\alpha=1$, the variation of numerical damping ratio against $\Omega$ with varying $\xi$ is shown in Figure 16(a). It can be seen that the numerical damping ratio is negative and its absolute value increases with higher $\Omega$ and $\xi$. The correlation of the numerical damping ratio versus $\Omega$ with varying $\alpha$ is plotted in Figure 16(b) for $\xi=2 \%$. It is clear that the numerical damping ratio is also negative for the assumed parameters. Furthermore, the absolute value of the numerical damping ratio increases as the accuracy of the estimated tangent stiffness decreases. A study of the variation of the numerical damping ratio versus $\Omega$ with varying $\beta$ showed that the absolute value of the
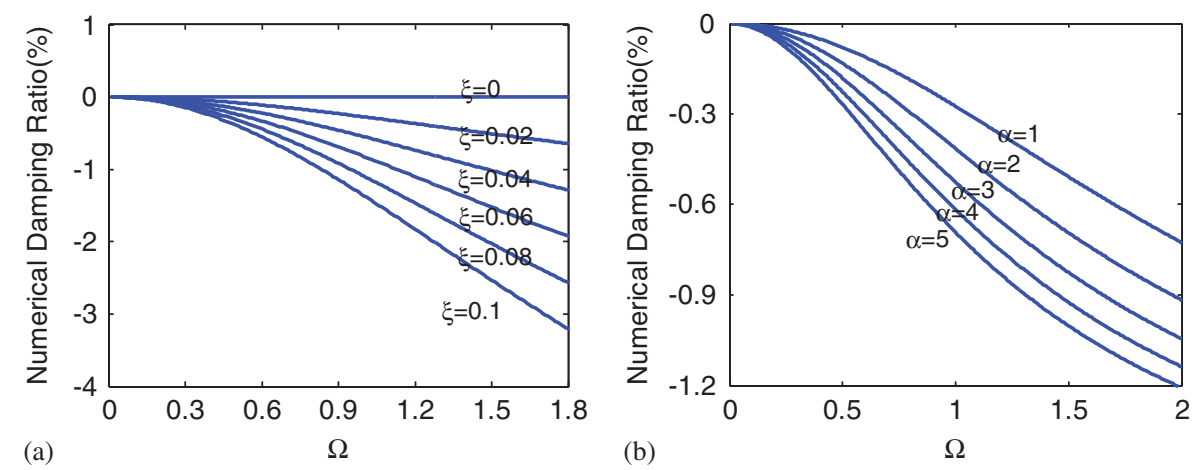

Figure 16. Trends in the numerical damping ratio: (a) $\Omega$ with varying $\xi$ and (b) $\Omega$ with varying $\alpha$. 

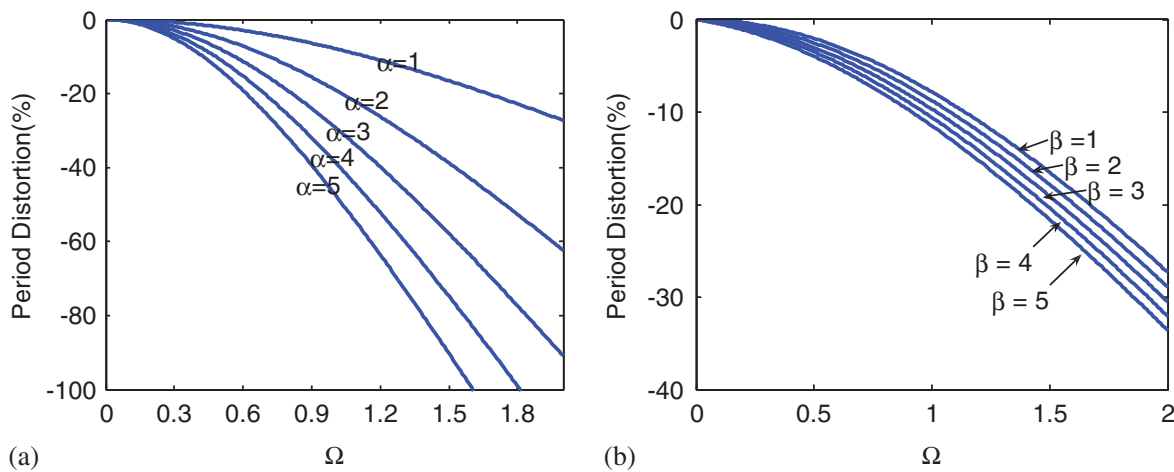

Figure 17. Trends in period distortion versus $\Omega$ : (a) $\Omega$ with varying $\alpha(\beta=1, \xi=0.02)$ and (b) $\Omega$ with varying $\beta(\alpha=1, \xi=0.02)$.

numerical damping ratio increases as $\beta$ becomes larger, but the difference between the results for varying $\beta$ is minor.

Period distortion can be expressed as

$$
\frac{\Delta T_{\mathrm{d}}}{T_{\mathrm{d}}}=\frac{T_{\mathrm{d}}-\bar{T}_{\mathrm{d}}}{T_{\mathrm{d}}}=1-\frac{\omega_{\mathrm{d}}}{\bar{\omega}_{\mathrm{d}}}=1-\frac{\Omega}{\bar{\Omega}} \sqrt{1-\xi^{2}}
$$

Period distortion against $\Omega$ with varying $\alpha$ is plotted in Figure 17 (a) for $\beta=1$ and $\xi=0.02$. It can be seen that the period extends for the prescribed parameters. However, distortion can be alleviated by increasing the accuracy of the estimated tangent stiffness. Using $\alpha=1$, and $\xi=0.02$, period distortion against $\Omega$ with varying $\beta$ is plotted in Figure $17(\mathrm{~b})$. It is shown that period distortion increases with increasing $\beta$. Nevertheless, the effect of $\beta$ on the period distortion is not significant. Finally, it can be observed from Equation (18) that the effect of varying $\xi$ on period distortion is not obvious since the damping ratios for most civil structures are less than $5 \%$.

\section{SUMMARY AND CONCLUSIONS}

A strategy for estimating the tangent stiffness of a system during hybrid simulation was proposed. The new method is based on the premise that the specimen's tangent stiffness does not change substantially during the few steps preceding the current time-integration step. By exploiting this assumption, it is possible to solve a set of equations (possibly over-determined) to identify the full stiffness matrix for a multi-dof test specimen. Although the proposed technique is general and can be applied to a variety of the implicit algorithms for time-stepping integration, it was combined in this work with the OSM resulting in a new operator-splitting formulation designated as OSM-US.

The capabilities, advantages and limitations of OSM-US were discussed and demonstrated through several examples of single and multi-dof specimens. The research results showed that the proposed algorithm provides results that are better than those produced via the regular OSM alone, especially for damped structures undergoing highly inelastic behavior during testing. The enhancement was observed in improved displacement calculations, reduced cumulative energy errors and lower unbalanced forces between substructures in the examples studied. The accuracy, 
stability and error propagation characteristics of the modified OSM were also studied theoretically as well as numerically. Specifically, the study quantified the effect of tangent stiffness estimation error on the stability and accuracy of a single dof model.

Although the OSM-US has shown much promise in the numerical simulations results presented in this paper, it is necessary to conduct physical tests to make sure that it would work under practical hybrid simulation conditions. Moreover, the developed method has been applied to structures with overall hardening response and dominated by first mode behavior. Additional research is necessary to investigate if it will provide similarly good results for softening structures and to develop it for use in structures with higher mode response.

\section{ACKNOWLEDGEMENTS}

The research described herein was sponsored in part by the National Science Foundation under grant No. CMS 0530383 and the University of Michigan. The authors would also like to acknowledge the extensive assistance in software operation and development provided by Dr O.S. Kwon at the University of Illinois at Urbana-Champaign. The opinions expressed in this paper are those of the authors and do not necessarily reflect the views of the sponsors or the individual mentioned here.

\section{REFERENCES}

1. Takanashi K, Udagawa K, Seki M, Okada T, Tanaka K. Earthquake response analysis of steel frames by computer-actuator on-line system. Proceedings of the 5th Japan Earthquake Engineering Symposium, Tokyo, Japan, November 1978; 1321-1328.

2. Takanashi K, Nakashima M. Japanese activities on on-line testing. Journal of Structural Engineering (ASCE) 1987; 113(7):1014-1032.

3. Shing PB, Mahin SA. Pseudodynamic test method for seismic performance evaluation: theory and implementation. Report No. UCB/EERC-84/12, Earthquake Engineering Research Center, University of California, Berkeley, 1984.

4. Combescure D, Pegon P. $\alpha$-Operator-splitting time integration technique for pseudodynamic testing error propagation analysis. Soil Dynamics and Earthquake Engineering 1997; 16(7-8):427-443.

5. Mahin SA, Shing PB. Pseudodynamic method for seismic testing. Journal of Structural Engineering (ASCE) 1985; 111(7):1482-1503.

6. Shing PB, Vannan MT, Cater E. Implicit time integration for pseudodynamic tests. Earthquake Engineering and Structural Dynamics 1991; 20:551-576.

7. Shing PB, Bursi OS, Vannan MT. Pseudodynamic tests of a concentrically braced frame using substructuring techniques. Journal of Constructional Steel Research 1994; 29:121-148.

8. Nakashima M, Kato H, Takaoka E. Development of real-time pseudo dynamic testing. Earthquake Engineering and Structural Dynamics 1992; 21:79-92.

9. Nakashima M, Masaoka N. Real-time on-line test for MDOF systems. Earthquake Engineering and Structural Dynamics 1999; 28:393-420.

10. Wu B, Bao H, Ou J, Tian S. Stability and accuracy analysis of central difference method for real-time substructure testing. Earthquake Engineering and Structural Dynamics 2005; 34:705-718.

11. Molina FJ, Verzeletti G, Magonette G, Buchet P, Geradin M. Bi-directional pseudodynamic test of a full-size three-storey building. Earthquake Engineering and Structural Dynamics 1999; 28:1541-1566.

12. Darby AP, Blakeborough A, Williams MS. Real-time substructure tests using hydraulic actuator. Journal of Engineering Mechanics (ASCE) 1999; 125(10):1133-1139.

13. Darby AP, Blakeborough A, Williams MS. Improved control algorithm for real-time substructure testing. Earthquake Engineering and Structural Dynamics 2001; 30:431-448.

14. Pan P, Tomofuji H, Wang T, Nakashima M, Ohsaki M, Mosalam K. Development of peer-to-peer (P2P) internet online hybrid test system. Earthquake Engineering and Structural Dynamics 2006; 35:867-890.

15. Thewalt CR, Mahin SA. An unconditionally stable hybrid pseudodynamic algorithm. Earthquake Engineering and Structural Dynamics 1994; 24:723-731. 
16. Nakashima M, Masaoka N. Real-time on-line test for MDOF systems. Earthquake Engineering and Structural Dynamics 1999; 28(4):393-420.

17. Zhang Y, Sause R, Ricles JM, Naito CJ. Modified predictor-corrector numerical scheme for real-time pseudo dynamic tests using state-space formulation. Earthquake Engineering and Structural Dynamics 2005; 34:271-288.

18. Thewalt CR, Mahin SA. Hybrid solution techniques for generalized pseudodynamic testing. Report No. $U C B / E E R C-87 / 09$, Earthquake Engineering Research Center, University of California, Berkeley, 1987.

19. Nakashima M, Kato H. Experimental Error Growth Behavior and Error Growth Control in On-line Computer Test Control Method. Building Research Institute, Ministry of Construction, 1987.

20. Shing PB, Mahin SA. Experimental error propagation in pseudodynamic testing. Report No. UCB/EERC-83/12, Earthquake Engineering Research Center, University of California, Berkeley, 1983.

21. Shing PB, Mahin SA. Pseudodynamic test method for seismic performance evaluation: theory and implementation. Report No. UCB/EERC-84/12, Earthquake Engineering Research Center, University of California, Berkeley, 1984.

22. Hilber HM, Hughes TJR, Taylor RL. Improved numerical dissipation for time integration algorithm in structural dynamics. Earthquake Engineering and Structural Dynamics 1977; 5:283-292.

23. Shing PB, Vannan MT, Carter E. Implicit time integration for pseudodynamic tests. Earthquake Engineering and Structural Dynamics 1991; 20:551-576.

24. Shing PB, Vannan MT, Carter E. Evaluation of reinforced masonry shear wall components by pseudodynamic testing. Proceedings of the 4th U.S. National Conference on Earthquake Engineering, Palm Springs, CA, 1990; 829-838.

25. Nakashima M, Kaminosono T, Ishida M, Ando K. Integration techniques for substructure pseudo dynamic test. Proceedings of the 4th U.S. National Conference on Earthquake Engineering, Palm Springs, CA, 1990; 515-524.

26. Thewalt CR, Roman M. Performance parameters for pseudodynamic tests. Journal of Structural Engineering (ASCE) 1994; 120(9):2768-2781.

27. Nocedal J, Wright SJ. Numerical Optimization. Springer: New York, 1999. ISBN 0-387-98793-2.

28. Carrion JE, Spencer BF. Real-time hybrid testing using model-based delay compensation. Proceedings of the 4th International Conference on Earthquake Engineering, Taipei, Taiwan, 2006.

29. Broyden CG. A class of methods for solving nonlinear simultaneous equations. Mathematics of Computation 1965; 19:577-593.

30. MATLAB R2006a Help Manual, 2007.

31. OpenSees version 1.7 User Manual, Pacific Earthquake Engineering Research Center, University of California, Berkeley. Available from: http://opensees.berkeley.edu, 2006.

32. Shing PB, Mahin SA. Computational aspects of a seismic performance test method using on-line computer control. Earthquake Engineering and Structural Dynamics 1985; 13:507-526. 